\title{
Orientation selective 2D-SIFTER experiments at X-band frequencies
}

A. M. Bowen ${ }^{1,2^{*}}$, N. Erlenbach ${ }^{1 *}$, P. van Os ${ }^{1}$, L. S. Stelzl $^{3}$, S. Th. Sigurdsson ${ }^{4}$, and T. F. Prisner ${ }^{1}$

${ }^{1}$ Institute of Physical and Theoretical Chemistry and Center of Biomolecular Magnetic Resonance, Goethe University, Max-von-Laue-Str. 7, 60438 Frankfurt am Main, Germany.

${ }^{2}$ Current address: Centre for Applied Electron Spin Resonance, Inorganic Chemistry Laboratory, Department of Chemistry, University of Oxford, South Parks Road, Oxford OX1 3QR, U.K

${ }^{3}$ Department of Theoretical Biophysics, Max Planck Institute of Biophysics, 60438 Frankfurt am Main, Germany

${ }^{4}$ Department of Chemistry, Science Institute, University of Iceland, Dunhaga 3, 107 Reykjavik, Iceland

E-mail: prisner@chemie.uni-frankfurt.de

*Both authors contributed equally to this work

\section{Abstract}

Frequency-correlated 2D SIFTER with broadband pulses at X-band frequencies can be used to determine the inter-spin distance and relative orientation of nitroxide moieties in macromolecules when the flexibility of the spin-labels is restricted. At X-band frequencies the EPR spectrum of nitroxides is governed by the strongly anisotropic nitrogen hyperfine coupling. For rigid spin-labels, where the orientation of the inter-connecting vector $\boldsymbol{R}$ correlates to the relative orientations of the nitroxide labels, the dipolar oscillation frequency varies over the EPR spectral line shape. Broadband shaped pulses allow excitation of the complete nitroxide EPR spectra. In thi case, Fourier transform of the echo signal gives both fast and direct access to the orientation dependent dipolar coupling. This allows determination of not only the inter-spin distance $R$, but also their mutual orientation. Here we show the application of the frequency-correlated 2D SIFTER experiment with broadband pulses to a bis-nitroxide model compound and to a double stranded DNA sample. In both molecules, there is restricted internal mobility of the two spin-labels. The experimental results are compared to orientation selective PELDOR experiments and simulations based on a simple geometrical model or MD simulations describing the conformational flexibility of the molecules. Fourier transformation of the SIFTER echo signal yields orientation selective dipolar time traces over the complete EPR-spectral range. This leads to an improved frequency resolution and either to a reduced experimental measurement time or a larger span of frequency offsets measured compared to orientation selective PELDOR experiments. The experimental potential and limitations of the 2D SIFTER method for samples containing rigid spin-labels will be discussed. 


\section{Introduction}

Pulsed Dipolar Spectroscopy (PDS) methods measure the dipolar interaction between unpaired electron spins. It is an increasingly important set of techniques in biophysics for the determination of structure and structural flexibility in biomolecules. Example of PDS techniques include: Pulsed Electron Double Resonance (PELDOR [1, 2], also known as Double Electron-Electron Resonance, DEER) [3, 4], Double Quantum Coherence (DQC) [5], Relaxation Induced Dipolar Modulation Enhancement (RIDME) [6, 7], and the Single Frequency Technique for Refocusing dipolar couplings (SIFTER) [8]. All PDS techniques result in oscillating time traces, where the frequency of oscillation $\omega_{d d}$ can be related to the inter-spin distance $R$ and, in cases of rigid radical moieties or spin-labels, to the orientation $\theta$ of the distance vector $\boldsymbol{R}$ with respect to the external magnetic field direction.

$$
\omega_{\mathrm{dd}}=\frac{\mu_{0} \mu_{B}^{2}}{4 \pi \hbar} g_{A} g_{B} \frac{1-3 \cos ^{2} \theta}{R^{3}}
$$

Here $\mu_{0}$ is the magnetic susceptibility of a vacuum, $\mu_{B}$ is the Bohr magneton and $\hbar$ is the reduced Planck constant. $g_{A}$ and $g_{B}$ are the effective g-factors of the two coupled spins (notated A and B).

Many biomolecules are inherently EPR silent; therefore, in order to study them spin-labels must be used. Frequently, these spin-labels are nitroxide based, due to their stability and ease of handling. In the case, that the nitroxide spin-labels are attached to the biomolecule via flexible tethers, all orientations of $\boldsymbol{R}$ with respect to the external magnetic field (described by the angle $\theta$ ) contribute to each resonance position within the EPR spectra. This is typically the case for the MTSSL (1-oxyl-2,2,5,5-tetramethylpyrroline-3-methyl methanethiosulfonate) spin-label covalently bound to cysteins of proteins, modified via site directed mutagenesis [9]. In this case, the Fourier transform of the dipolar time trace results in the well-known Pake pattern. This can be analysed purely in terms of the distance $R$ between both spin-labels or a distance distribution $P(R)$ for flexible macromolecules [10].

In contrast, when rigid nitroxides are attached to the macromolecule such that they have limited motion, as is the case for the spin-labeled DNA molecule presented in this work, different angles $\theta$ contribute to the dipolar time trace, depending on the frequencies excited in the EPR spectrum. Currently, the most common method to probe orientation selection in PDS is 2D-PELDOR, a method where several one-dimensional time traces are recorded sequentially by varying the pump and/or detection pulse frequency or the external magnetic field strength to sample different orientations of the spin-label with respect to the external magnetic field. Commonly, for nitroxide spin-labels at X-band, this is achieved by positioning the pump pulse at the centre of the nitroxide spectrum and varying the detection pulse frequency offset. 
For nitroxide spin-labels, when the system is fully rigid, a set of orientation selective X-band data may allow full determination of the distance and relative orientation between both spin-labels [11]. However, as most molecules have some degree of flexibility, an increased set of orientation selective PDS traces may provide a more stable solution.

A disadvantage of the 2D PELDOR methodology is that a number of different time traces must be collected to allow accurate analysis making the experiment time consuming. Furthermore, to sample the full range of orientations at X-band frequencies, the probe frequencies have to be offset up to $90 \mathrm{MHz}$, which due to the finite resonator bandwidth leads to varying microwave field strengths as a function of the offset frequency. This can lead to a variation of the excitation bandwidths and modulation depths as a function of the pump and probe frequencies. These experimental variations make the quantitative analysis of such 2D-PELDOR experiments more demanding.

For molecules with rigid spin-labels attached, it is therefore highly desirable to have a single two-dimensional experiment in which the complete spectrally resolved dipolar time traces can be detected simultaneously. This can be achieved by single frequency PDS methods, such as DQC and SIFTER, if the pulses that are used excite the whole nitroxide EPR spectrum, as already realized by Jack Freed in several very elegant and benchmarking experiments [12-14].

Whereas rectangular pulses need very high microwave field strengths and short pulse lengths to excite the whole nitroxide spectrum, broadband pulses shaped by a fast Arbitrary Waveform Generator (AWG) can achieve such excitation bandwidths with more moderate microwave powers for prolonged pulse lengths $[15,16]$. We have shown that such broadband pulses improve the quality of the 1D SIFTER pulse sequence considerably [16]. However, in the one-dimensional form, integrating over the echo signal has the effect of removing all orientation information [17]. Doll and Jeschke reported a frequency-correlated 2D-SIFTER recorded at Q-band frequencies (35 GHz) using broadband chirp pulses [18].

In this manuscript, we present two-dimensional frequency correlated 2D-SIFTER performed at X-band frequencies, where the spectrum asymmetry is governed by the hyperfine anisotropy and orientation selection is more easily probed compared to Q-band frequencies, where the g-tensor and nitrogen hyperfine asymmetries have similar size, and without a wide bandwidth resonator it is often not possible to sample sufficiently large offsets to adequately sample the orientation selectivity in PDS techniques. We compare the 2D-SIFTER experiments with PELDOR experiments performed with several probe frequencies for a bis-nitroxide chemical compound and a double stranded DNA molecule with two rigid Ç spin-labels [19-22]. The optimization of the broadband SIFTER pulse sequence for orientation selective 2D experiments is described, and to validate the 
methodology, we show comparison of the frequency correlated 2D-SIFTER data recorded to the current gold standard of orientation selective PELDOR and explain the differences in the forms of the data. Finally, we show that it is possible to quantitatively simulate the spectrally resolved time traces using an analytical methodology [11] and compare the data with simulations based on geometrical models [21, 23] or MD simulations [24, 25].

\section{Experimental Methods:}

\section{Instrumentation and experimental conditions}

Experiments were performed at $\mathrm{X}$ band $(9.5 \mathrm{GHz})$ on a commercially available Bruker Elexsys E580 spectrometer. For experiments using broadband pulses this was modified with an ITS 8200 (Innovative Technical Systems) Arbitrary Waveform Generator (AWG) positioned before the $1 \mathrm{~kW}$ Travelling Wave Tube (TWT) amplifier as described in [16]. An Oxford Instruments CF935 cryostat was used to cool the sample and resonator, a Bruker MS3 3mm loop gap resonator. All experiments were performed at 50K using a continuous flow of liquid helium and the temperature maintained using an Oxford Instruments ITC 503 temperature control unit.

Pulsed electron double resonance experiments used the four pulse DEER sequence [3, 4], with detection pulse lengths of $32 \mathrm{~ns}$ and pump pulse lengths of $16 \mathrm{~ns}$. In all cases, the pump pulse frequency was set to be resonant with the maximum of the nitroxide spectrum and the detection pulses were offset from this frequency by 30 or $40 \mathrm{MHz}$ to 80 or $90 \mathrm{MHz}$ in $10 \mathrm{MHz}$ increments. The bandwidth of the $16 \mathrm{~ns}$ pump pulse is sufficient to excite the complete central hyperfine transition $\left(\mathrm{m}_{\mathrm{I}}=0\right)$ of the nitroxide spectrum, and as a result excites all of the orientations of the nitroxide centre with respect to the magnetic field in this transition while exciting minimal amounts of the other $A_{z}$ transitions. This results in a pump pulse that yields very little orientation selection. The frequency offsets of 30 to $90 \mathrm{MHz}$ for the detection frequency were used as this allowed detection on the more intense $A_{z}, m_{I}=+1$ feature; a similar set of traces could be measured on the $A_{z}, m_{I}=-1$ feature with negative frequency offset between the pump and detection frequencies however this would yield traces of lower signal to noise. The lower limit of $30 \mathrm{MHz}$ corresponds to the frequency at which overlap of the pump and detection pulses becomes significant and the upper limit of $80-90 \mathrm{MHz}$ to the edge of the nitroxide spectrum.

To reduce the presence of nuclear modulations in the time traces a tau-averaging procedure of 8 steps with an increment of $56 \mathrm{~ns}$ (for deuterated solvent samples) were utilized. For the chemical model system in total 540 time steps with 640 averages per data point have been recorded, resulting in a total acquisition time of about 45 minutes per offset. The echo signal is integrated over a 32 ns time window. In the DNA system, the 
concentration of the sample was lower. Therefore, in order to detect a reasonable signal to noise ratio (SNR), the number of averages was varied for different pump pulse offsets.

The set of PELDOR experiments recorded on the DNA system were recorded keeping the pump pulse frequency constant at the centre of the resonator and the detection pulses were retuned at a different frequency offset for each subsequent experiment. This makes recording the data labour-intensive as retuning is required between each experiment. For the bis-nitroxide model compound an alternative approach was used, whereby the detection frequency was kept constant and the pump frequency and attenuation changed between each experiment. Pulse length and field strength of the pump pulse were calibrated for each offset frequency of the overcoupled MS3 resonator to achieve same excitation efficiency for all PELDOR traces. This has been reliably achieved, as observed in the data in Figure 2 and Figure 3 where the modulation depths were in good agreement with the simulations using experimental methods.

For SIFTER we used broadband $\operatorname{sech}(\mathrm{x}) / \tanh (\mathrm{x})$ pulses [26] combined with a WURST, (wideband, uniform rate, smooth truncation) amplitude function [27], as previously employed [16]. Two forms of the experimental sequence using broadband pulses were trialled; one where all pulses had equal length (200 ns) and chirp rate, resulting in the relative amplitude of the pulses being ca. 0.4:1 for the $\pi / 2(200 \mathrm{~ns}): \pi(200 \mathrm{~ns})$ pulses; and another where the pulse length of the final $\pi$ pulse used was $100 \mathrm{~ns}$. The second sequence has the effect of refocusing the phase of all components of the echo at a fixed time [16], leading to the largest solid echo signal. However, it was found that using pulses of equal lengths (200 ns) yielded a better modulation depth, as the longer pulses possessed better excitation efficiency. All of the pulses were calibrated to have bandwidths of ca. $200 \mathrm{MHz}$, (sufficient to excite the complete nitroxide spectrum). In this optimised sequence the $200 \mathrm{~ns} \pi$ pulses used in the experiment had $\beta=9.3 \mathrm{MHz}$, bandwidth parameter $=0.017 \mathrm{x} 2 \pi, \omega_{1, \max }=25 \mathrm{MHz}, \varphi=0, \beta_{\text {WURST }}=16$ $\mathrm{MHz}, n=16$. The $200 \mathrm{~ns} \pi / 2$ pulses used had $\beta=9.3 \mathrm{MHz}$, bandwidth parameter $=0.017 \mathrm{x} 2 \pi, \omega_{1, \max }=10 \mathrm{MHz}$, $\varphi=0, \beta_{\text {WURST }}=16 \mathrm{MHz}, n=16$. For optimum performance, the bandwidth of the broadband pulses was set symmetrically to the edges of the nitroxide EPR spectrum, and thus the carrier frequency was offset from the maximum of the nitroxide spectrum. For our data analysis, a frequency dependent phasing of each time trace after Fourier transform of the echo was used to minimise the contributions in the imaginary channel.

SIFTER was recorded both as a one-dimensional experiment, which allows determination of the distance $R$ by Tikhonov regularization, and as a two-dimensional one to enable orientation dependent analysis. As with the PELDOR sequence described above tau-averaging was incorporated in all of the SIFTER experiments by 
increasing each tau period by 56 ns over 8 steps to average over the deuterium nuclear oscillations form the deuterated solvents used.

\section{Processing of experimental data}

For PELDOR time traces an exponential background correction was performed using DeerAnalysis to remove the background due to intermolecular dipolar interactions [10]. SIFTER backgrounds are known to contain components due to both inter molecular dipolar interactions and relaxation [29]. It is possible to remove the part due to relaxation by recording an additional experimental SIFTER traces under the same conditions with the second $\pi / 2$ pulse removed [29]. The two systems presented here were optimised to maximise modulation depth and contributions from relaxation were minimized using deuterated solvents. This means that the data did not require any background correction.

In frequency correlated 2D-SIFTER the raw echo transients stored from the two dimensional experiments were apodized by a Gaussian function centred at the centre of the echo in the direct time domain to supress the noise at the edge of the echo transient. These data were then Fourier transformed in the direct time domain. After that the time traces for each offset frequency were phased individually using a $0^{\text {th }}$ order correction to achieve a maximum signal in the real part at the time point $\tau_{1}-\tau_{2}=0$.

\section{Samples}

Data were recorded on two samples: a bis-nitroxide model-compound [16] prepared at a concentration of $150 \mu \mathrm{M}$ in deuterated toluene and a double stranded DNA molecule with two rigid spin-labels Ç at position 3 and 15 prepared in a deuterated solvent [21]. 
a)

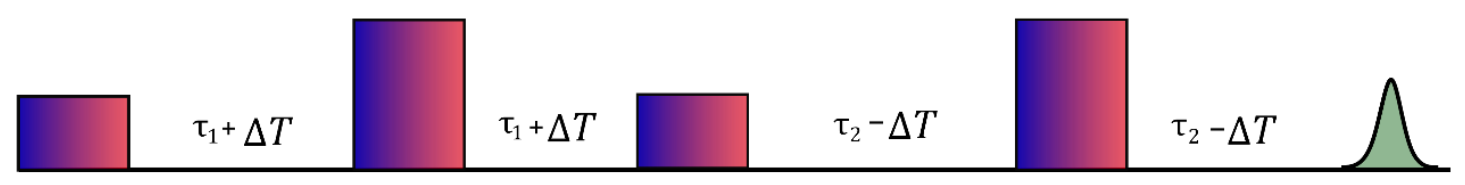

b)<smiles>CCCCCCc1cc(C#Cc2ccc(-c3ccc(C#CC4=CC(C)(C)N([O])C4(C)C)cc3)cc2)c(CCC)cc1C#Cc1ccc(-c2ccc(C#CC3=CC(C)(C)N([O])C3(C)C)cc2)cc1</smiles>

c)

\section{5' - GT ÇAG TGC GCG CGC GCG ATC - 3' 3' - CA CTC ACG CGC GCG ÇGC TUG - 5'}

d)

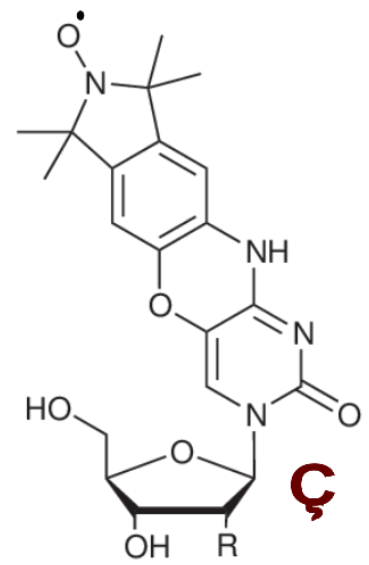

Figure 1: a) Broadband SIFTER pulse sequence with all pulses of equal length here. b) Bis-nitroxide model compound used in this study. c) Sequence of the dsDNA molecule used in this study. d) Chemical structure of the cytidine analogue spin-label Ç incorporated into the dsDNA molecule.

\section{Simulation Methods}

The simulation procedure of orientation dependent PELDOR time traces, based on the secular approximation of the dipolar interaction (formula 1), is described in detail in [11, 22]. This assumes that the dipolar coupling strength is smaller than the offset of pump and probe frequency in the PELDOR experiments. This approximation is not fulfilled for all possible spin-pairs in single-frequency dipolar experiments, such as DQC or SIFTER [28], especially for very short distances $(<1.5 \mathrm{~nm})$. The inter-spin distances in the two molecules used here are longer than $2 \mathrm{~nm}$ and the excellent agreement of the experimental SIFTER data with the simulations and PELDOR data show that the approximation is still sufficient here. 
For the analytical simulations of the PELDOR and SIFTER dipolar time traces of the bis-nitroxide compound, the relative positions of the nitroxide $\mathrm{N}-\mathrm{O}$ moieties and their conformational distribution is required. A geometric model was used, as described in [21] to provide this data. For the ds-DNA molecule, the MD simulation, detailed in [25], was directly used to determine the distances and relative orientations between the two Ç spin-labels for the quantitative simulation of the PELDOR and SIFTER time traces [23].

\section{Experimental Results}

\section{Optimization of Pulse Settings for 2D-SIFTER}

PDS experiments with rigid spin-labels can encode up to six parameters: the distance $R$, the three Euler angles describing the mutual orientation between both nitroxide spin-labels and the two polar angles describing the orientation of the $\boldsymbol{R}$ vector with respect to one of the nitroxides. However, when the pump is orientation unselective, as is the case for PELDOR at X-band with a pump pulse that excited the complete central transition and frequency correlated SIFTER, only the distance and polar angles of each of the centres can be resolved, and in the case that the spin-centres are chemically identical the number of angles that can be resolved is further reduced. To unravel the unknown distance and orientation parameters quantitatively an X-band PELDOR data set, the pump pulse positioned at the nitroxide maximum and with detection-pump frequency offsets ranging from 30-40 up to 80-90 MHz with $10 \mathrm{MHz}$ steps, can be used [22]. In a 2D frequency correlated technique, these frequencies are captured within a single experiment. The signal intensity of each dipolar trace will depend on the spectral intensity at each frequency, which is a feature of the radical species being studied, and on the degree of excitation at each frequency. The broadband pulses used in our experiments were designed to have a rectangular frequency profile across the whole EPR spectrum of the nitroxide. Therefore, the Fourier transform of the echo for zero dipolar evolution time should reproduce the EPR spectrum. Experimentally it was found that the measured profile did not always agree with expected nitroxide profile measured via an echo detected field sweep. There are several reasons for this: Firstly, the exact shape of the Fourier transform will depend on the signal processing used; if a Gaussian apodization window is used this improves SNR but may cause some broadening of the resulting spectrum. Secondly, the precise shape of the echo detected field sweep depends on the lengths of the pulses used, the excitation bandwidths of these pulses and the integration window used in detection. The broad bandwidth of short pulses can cause broadening of the spectrum from its expected form when the integration window used is less than ca. $200 \mathrm{~ns}$; in all data sets recorded using rectangular time domain pulses the integration window used was set to the length of the $\pi$ pulse. Finally, although the broadband pulses were designed to have equal excitation at all frequencies of the nitroxide spectrum, convolution of the pulses 
with the resonator bandwidth and the bandwidths of other components used in generation of the pulses and detection may cause a distortion of the spectrum observed. Specifically the video amplifier in the Bruker E580 has a bandwidth of only $200 \mathrm{MHz}$. Experiments conducted with the amplifier removed, while showing slight increase in the amplitudes of the signals at large frequency offsets from the carrier frequency did not show differences in the shape of the dipolar traces recorded at these offsets. While it is possible to compensate for the component bandwidths using compensated pulses [29], as our analysis is based on the shape of the traces at different offsets, rather than the signal amplitude, this was not necessary for the experiments presented in this work. Furthermore, the experiments were conducted with the video amplifier in use, as the overall signal to noise was marginally increased. Artefacts due to carrier frequency leakage in the pulse up-conversion process can also occur and show up in the frequency domain as sharp spikes. Although all these features may distort the frequency spectrum at zero dipolar evolution time of the experiment, they do not change the observed dipolar oscillations in the indirect time domain (although they can degrade the signal to noise of the traces at specific frequencies).

\section{Comparison of simulations with the experimental data.}

For a quantitative analytical simulation, the following spin parameters were used: $g=(2.0088,2.0065,2.0027)$ and nitrogen hyperfine coupling of $A[M H z]=(15,15,95)$. Orientationselective PELDOR data were simulated using the experimental pulse lengths, field strengths and offset frequencies. The conformational model for the DNA system is based on a molecular dynamics simulation [25], and the conformers for the bis-nitroxide model compound were derived from a conical model simulating the allowed rotation of the nitroxide moieties and the bending of the linker unit as described before [11,20,23]. We achieved very good agreement between simulated and experimentally recorded PELDOR data sets for both molecules as demonstrated in Figure and Figure 3; bottom left plots. 
a

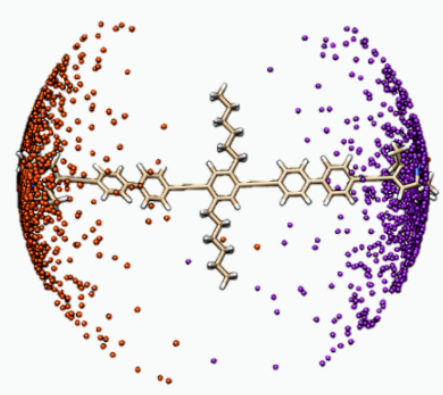

C

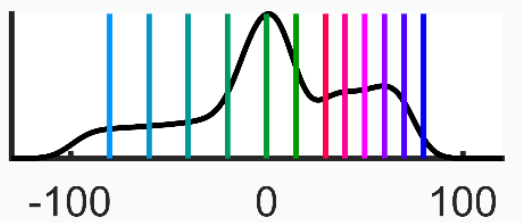

d offset $[\mathrm{MHz}]$

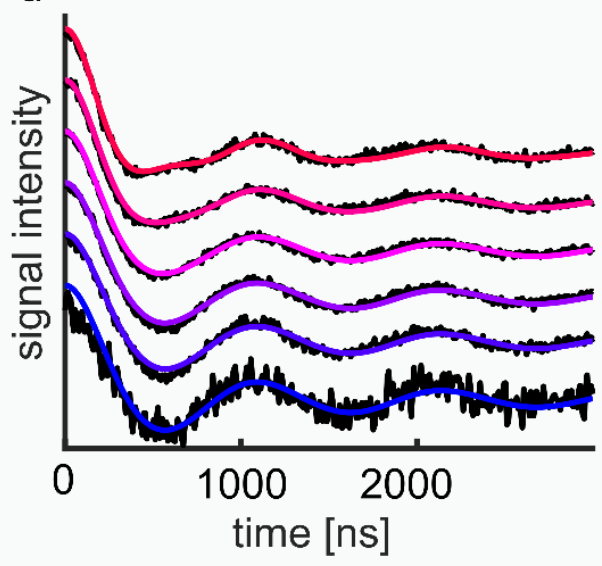

b

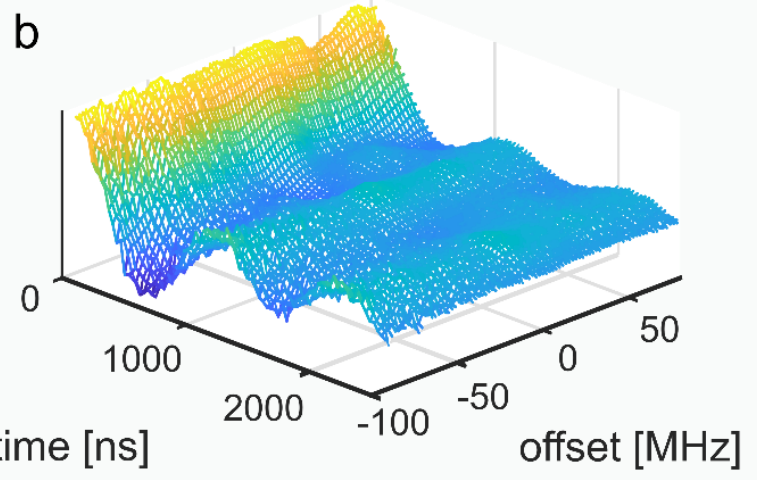

time [ns]

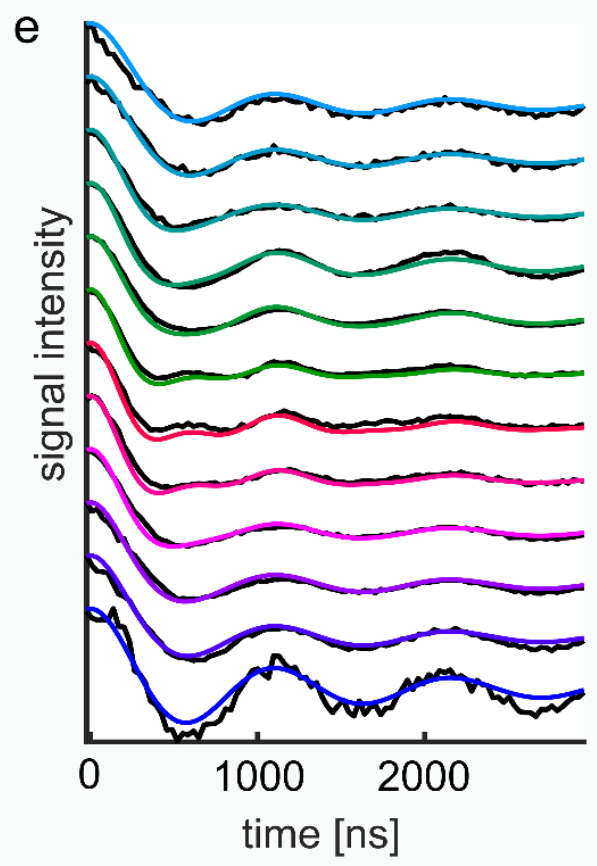

Figure 2: Results for bis-nitroxide model system: a) Spin-label orientations calculated using a cone model. b) Normalised frequency correlated SIFTER dataset measured with all pulses $200 \mathrm{~ns}$ long presented in the time domain of the dipolar dimension; time is $\tau_{1}-\tau_{2}$. c) Echo detected field sweep of the system presented in the frequency domain relative to the spectral maxima with coloured lines showing the detection positions offsets for the PELDOR data presented in d) and the frequency slice of the frequency correlated SIFTER data presented in e). d) PELDOR time traces, experimental data (black lines) with corresponding simulations (coloured lines). e) Slices from the frequency correlated 2D-SIFTER experiment (black lines) presented in b) with corresponding simulations (coloured lines). Molecular graphics and analyses were performed with the UCSF Chimera package. 

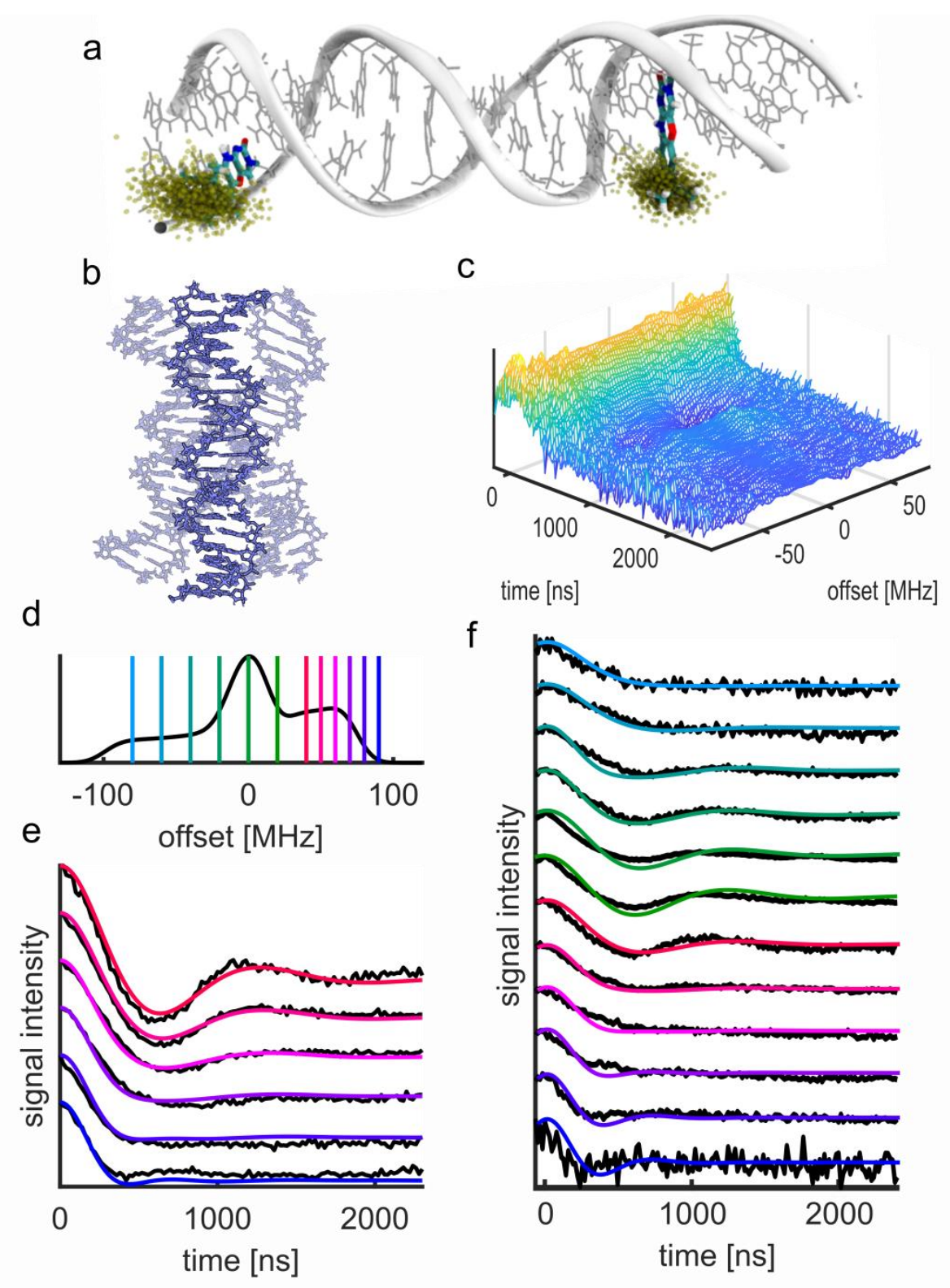

Figure 3: Results for the ds-DNA molecule: a) Spin-label orientations from molecular dynamic (MD) simulations of DNA(1,13), Ç structure from final frame from the MD trajectories shown as a stick representation relative to a ribbon representation of the DNA backbone. The positions of nitroxide spins over the course of a $200 \mathrm{~ns}$ MD trajectory are visualized as yellow spheres, showing 1000 spin positions per label, a subset of the 2000 orientations used in the simulation of the PDS traces. The distribution of the nitroxide spins results from the flexibility of the DNA backbone as shown with superimposed frames from the MD simulations in b). c) Normalised frequency correlated SIFTER dataset presented in the time domain of the dipolar dimension; time is $\tau_{1}-\tau_{2}$. d) Echo detected field sweep of the system presented in the frequency domain relative to the spectral maxima with coloured lines showing the detection positions offsets for the PELDOR data presented in e) and the frequency slice of the frequency correlated SIFTER data presented in f). e) PELDOR time traces, experimental data (black lines) with corresponding simulations (coloured lines). f) Slices from the frequency correlated SIFTER experiment (black lines) presented in c) with corresponding simulations (coloured lines). 
Comparison of the orientation selective PELDOR data recorded with the pump pulse at the central maximum and the detection pulse offset by $30 \mathrm{MHz}$ to $80 \mathrm{MHz}$ with individual traces from the 2D SIFTER data sets that were recorded at equivalent offsets from the nitroxide maximum as the detection frequency in the comparative PELDOR experiment showed small but reproducible differences in the shape of the time traces (Figure 4). These differences can be assigned to the differences in the excitation profiles of the pulses used in the two different experiments. As previously mentioned the experimental setup used for the PELDOR experiments results in a pump pulse that provides little orientation selection and therefore the orientation selection is provided mainly by the detection pulses. Likewise in SIFTER the orientation selection is provided only by the detection. Thus a comparison of the SIFTER and PELDOR traces provides an insight into the degree of selectivity of the detection mechanism and how sensitive the orientation of the labels are to this. As the shape of the traces is sensitive to the orientation of the two labels, the degree of difference in the SIFTER and PELDOR traces detected at the same frequency offset from the nitroxide maximum is dependent on this and differs between the systems studied.

In the remainder of the paper offset frequency is defined for PELDOR as the offset of the detection frequency from the nitroxide central maximum, where the pump pulse is applied and in SIFTER experiments traces corresponding to the same offset frequency are classified as the frequency slice that has the same offset from the nitroxide maximum.
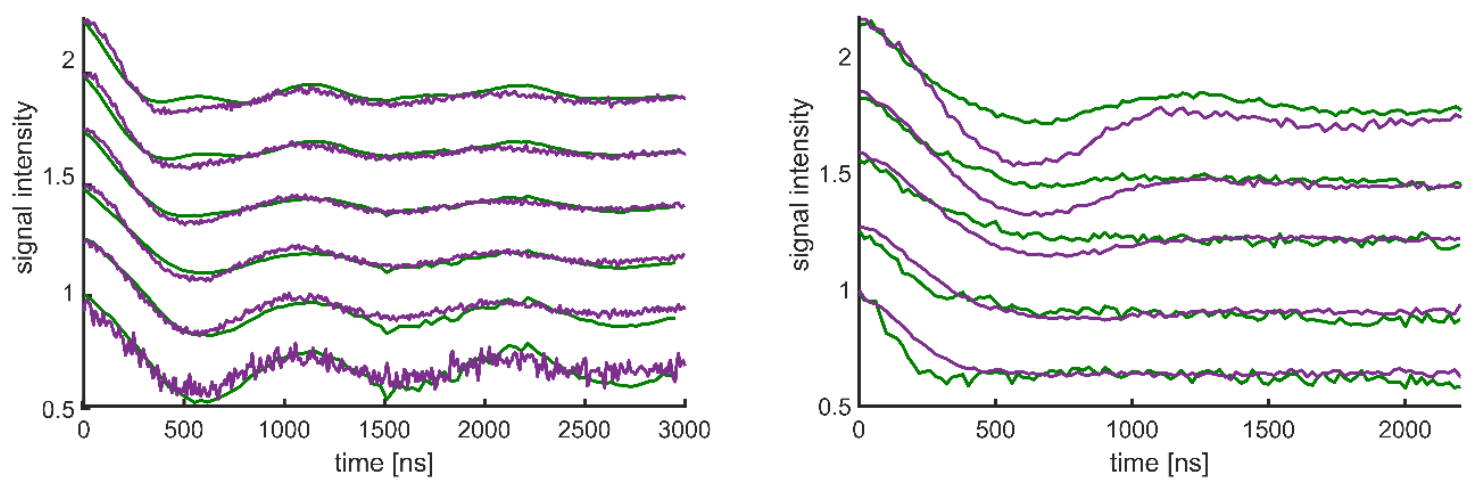

Figure 4: Comparison of PELDOR (violet) and SIFTER (green) time traces for the bis-nitroxide model system (on the left) and for the DNA (on the right). For the bis-nitroxide molecule the PELDOR time traces have offsets between pump and detection pulse frequency varying from $80 \mathrm{MHz}$ (lowest trace) to $30 \mathrm{MHz}$ (uppermost trace) in $10 \mathrm{MHz}$ steps. For the dsDNA molecule the PELDOR time traces have offsets between pump and detection pulse frequency varying from $80 \mathrm{MHz}$ (lower) to $40 \mathrm{MHz}$ (uppermost time trace ) in $10 \mathrm{MHz}$ steps. In both cases, the slices for the SIFTER time traces are taken at the same frequency offset from the signal maximum as the detection-pump frequency offset in the corresponding PELDOR time trace, where the pump pulse was applied to the signal maximum. 
In the broadband SIFTER experiment at X-band, all pulses are designed to excite the whole nitroxide EPR spectrum, as visible by the large experimental modulation depth. This can be analytically simulated by a pump pulse efficiency of $100 \%$. The 'detection pulse' bandwidth for the simulation of the 2D-SIFTER data was chosen as a Gaussian function to best fit the experimental frequency selective dipolar time traces obtained by Fourier transform of the SIFTER data set. The linewidth of $20 \mathrm{MHz}$ obtained from this fitting procedure is about a factor of 2 smaller than the excitation bandwidth of the 32 ns detection pulse sequence used for the PELDOR data set. It is limited by the inhomogeneous linewidth broadening from unresolved hyperfine couplings, which has to be used to simulate the nitroxide EPR spectral shape. Additionally, the Gaussian window function used for filtering of the echo transient before Fourier transformation will add further broadening. Correspondingly, the orientation selection achieved with the 2D SIFTER experiment is higher compared to that observed in the PELDOR experiment, leading to more pronounced oscillations in some traces, specifically for the bis-nitroxide model system at 50 to $30 \mathrm{MHz}$ frequency offset. Taking the differences in excitation and detection bandwidth between both methods into account, it was possible to simulate the 2D SIFTER data quantitatively, as presented in Figure 2 and Figure 3 (right side).

It should be noted that to record data of comparable SNR significantly fewer averages (about a factor of 6) were needed for 1D SIFTER compared to PELDOR; this is a result of the fact that SIFTER allows both pumping and detection of the whole nitroxide spectrum. Although fewer averages are needed for 1D SIFTER the average time to acquire one average of a data point can be longer partly, partly because of the external triggering of sequence with the AWG pulses. Although the sequences for the 1D- and 2D SIFTER experiments are identical, the detection procedures are not. In the 1D SIFTER experiment the solid echo is integrated over the central $50 \mathrm{~ns}$ time region whereas in 2D SIFTER a time trace with the full echo signal was recorded with a 1 ns time resolution and 512 points in the echo dimension. Within the Bruker hard- and software, recording the complete echo transient takes longer than integrating over the echo and therefore the time to acquire one average of a data point is increased. Comparing the SNR of the PELDOR data recorded to that of the 2D SIFTER it was found that approximately 4 times the number of averages were required for the 2D SIFTER compared to a single PELDOR experiment to yield time traces of comparable SNR. However, the 2D SIFTER experiment produces directly 100 individual time traces with a frequency spacing of approximately $2 \mathrm{MHz}$ distributed over the whole EPR spectrum. Our analysis additionally showed that the bandwidth of contributing frequencies to each time trace of the 2D SIFTER data set is significantly narrower compared to the PELDOR time traces, where the bandwidth is determined by the strength of the detection pulses. Therefore, significantly higher SNR can be achieved with the 2D SIFTER method with the same number of acquisitions. While hard- and software limitations might reduce 
this gain if the overall measurement time is considered, it may be possible to overcome some of these limitations on homebuilt spectrometers built to a different design and specification.

\section{Conclusions and Outlook}

In this work, we have shown that it is possible to record a $2 \mathrm{D}$ frequency correlated SIFTER spectrum with broadband pulses at X-band frequencies on a bis-nitroxide model compound and a ds-DNA molecule containing two rigid Ç spin-labels. This could be achieved using broadband excitation pulses created by an AWG and implemented into a commercial spectrometer and resonator. For rigid spin-labels, this technique allows orientation selective datasets to be acquired reliably from a single 2D experiment in a reasonable amount of time. As the information provided by both orientation dependent PELDOR traces and 2D SIFTER is comparable the question arises as to which is the better method to use. We have shown that the spectral resolution of such frequency correlated 2D-SIFTER time traces is superior to datasets achieved by the PELDOR method with several probe frequencies under typical conditions. For this reason when the sample being studied has some flexibility, fitting to the complete 2D SIFTER dataset may be preferable to a collection of frequency offset dependent PELDOR traces as the 2D SIFTER provides a more complete and finer grain description of the dipolar orientation dependence. However, the offset dependent modulation depth of the 2D PELDOR data set, which also contains information on the geometry between both spin-labels, is lost in the 1D SIFTER experiment. Based on our datasets it appears that approximately the same SNR is reached for a single time trace of the 2D SIFTER experiment compared to a PELDOR time trace with the same offset frequency (for the same number of averages per time step). However, the 2D SIFTER experiment can access all offsets over the full spectral range simultaneously with much higher spectral resolution compared to the PELDOR experiment. Integration of the time traces over all offsets (as achieved also with the 1D SIFTER experiment) allows also a simple determination of the distance $\mathrm{R}$ and the distance distribution function $\mathrm{P}(\mathrm{R})$ for samples with orientation selection [14].

We have also shown that the 2D-SIFTER data can be quantitatively simulated by the same analytical procedure used for the simulation of the orientation selective PELDOR time traces, taking the different excitation and detection bandwidth of both experiments into account. This is demonstrated here for a bis-nitroxide model compound, where the conformational ensemble of the spin system is obtained from a geometrical model, and for a double spin-labelled DNA molecule, where the conformational ensemble is derived from MD simulations. We believe that the frequency correlated 2D-SIFTER method with shaped broadband pulses is a very promising 
technique for the determination of structure and conformational flexibilities of macromolecules with rigid spinlabels at X-band frequencies.

\section{Notes}

\section{Acknowledgements}

We acknowledge financial support from the German Research Foundation (CRC902: Molecular Principles of RNA Based Regulation and SPP1601: New Frontiers in Sensitivity for EPR Spectroscopy). L.S. S is grateful for support from the Max Planck Society. A.M.B was supported by the Marie Curie GOIN program, and gratefully acknowledges her current fellowship support from the Royal Society and EPSRC for a Dorothy Hodgkin Fellowship (DH160004). We thank Dr. Plackmeyer for the synthesis of the bis-nitroxide model compound.

\section{References}

1. A. D. Milov, K. Salikhov and M. Shchirov, Sov. Phys. Solid State 23, 975 (1981).

2. A. D. Milov, A. Ponomarev and Y. Tsvetkov, Chem. Phys. Lett., 110, 67 (1984)

3. R. Martin, M. Pannier, F.Diederich, V. Gramlich, M. Hubrich and H. W. Spiess, Angw. Chemie Int. Ed., 37, 2833 (1998)

4. M. Pannier, S. Veit, A. Godt, G. Jeschke and H. W. Spiess, J. Magn. Reson. 142, 331 (2000).

5. P. P. Borbat and J. H. Freed, Chem. Phys. Lett. 313, 145 (1999).

6. L. V. Kulik, S. A. Dzuba, I. A. Grigoryev and Y. D. Tsvetkov, Chem. Phys. Lett. 343, 315 (2001).

7. S. Milikisyants, E. J. J. Groenen and M. Huber, J. Magn. Reson. 192, 275 (2008).

8. G. Jeschke, M. Pannier, A. Godt and H. W. Spiess, Chem. Phys. Lett. 331, 243 (2000).

9. C. Altenbach, T. Marti, H. Khorana and W. Hubbell, Science (80-. ). 248, 1088 (1990).

10. G. Jeschke, V. Chechik, P. Ionita, A. Godt, H. Zimmermann, J. Banham, C. R. Timmel, D. Hilger and H. Jung, Appl. Magn. Reson. 30, 473 (2006).

11. A. Marko and T. F. Prisner, Phys. Chem. Chem. Phys. 15, 619 (2013).

12. J. Gorcester and J. H. Freed, J. Chem. Phys. 88, 60 (1988).

13. S. Saxena and J. H. Freed, J. Phys. Chem. A101, 181 (1997)

14. P.P. Borbat and J. H. Freed Chapter 20 in eMagRes EPR Spectroscopy, edited by D. Goldfarb and S. Stoll, Wiley (2018)

15. A. Doll, S. Pribitzer, R. Tschaggelar and G. Jeschke, J. Magn. Res., 230, 27 (2013)

16. P. Schöps, P. E. Spindler, A. Marko and T. F. Prisner, J. Magn. Reson. 250, 55 (2015).

17. G. E. Merz, P. P. Borbat, A. J. Pratt, E. D. Getzoff, J. H. Freed and B. R. Crane, Biophys. J. 107, 1669 (2014).

18. A. Doll and G. Jeschke, Phys. Chem. Chem. Phys., 18, 33 (2016).

19. P. Cekan, A. L. Smith, N. Barhate, B. H. Robinson and S. T. Sigurdsson, Nucleic Acids Res. 36, 5946 (2008).

20. O. Schiemann, P. Cekan, D. Margraf, T. F. Prisner and S. T. Sigurdsson, Angew. Chemie - Int. Ed. 48, 3292 (2009).

21. A. Marko, V. Denysenkov, D. Margraf, P. Cekan, O. Schiemann, S. T. Sigurdsson and T. F. Prisner, J. Am. Chem. Soc. 133, 13375 (2011).

22. A. Marko, S. T. Sigurdsson and T. F. Prisner J. Magn. Res., 252, 187 (2015)

23. D. Margraf, B. Bode, A. Marko, O. Schiemann and T. F. Prisner, Mol. Phys., 105, 15 (2007)

24. A. Marko, D. Margraf, H. Yu, Y. Mu, G. Stock and T. F. Prisner. J. Phys. Chem., 130, 6 (2009)

25. L. S. Stelzl, N. Erlenbach, M. Heinz, T. F. Prisner and G. Hummer, J. Am. Chem. Soc., 30, 139 (2017).

26. M. S. Silver, R. I. Joseph and D. I. Hoult, Phys. Rev. A 31, 2753 (1985).

27. E. Kupce and R. Freeman, J. Magn. Reson. A 117, 246 (1995).

28. S. K. Misra, P. P. Borbat and J. H. Freed Appl Magn Reson 36, 237 (2008). 
29. P. E. Spindler, P. Schöps, A. M. Bowen, B. Endeward and T. F. Prisner, in eMagRes (John Wiley \& Sons, Ltd, Chichester, UK, 2016), pp. 1477-1492. 